\title{
Patient-specific meta-analysis of 12 -gene colon cancer recurrence score validation studies for recurrence risk assessment after surgery with or without 5FU and oxaliplatin
}

\author{
Greg Yothers ${ }^{1} \wedge$, Alan P. Venook ${ }^{2} \wedge$, Eiji Oki ${ }^{3}$, Donna Niedzwiecki ${ }^{4} \wedge$, Yan Lin $^{1} \wedge$, Michael R. Crager $^{5} \wedge$, \\ ${\text { Calvin } \mathrm{Chao}^{6 \wedge} \text {, Frederick L. Baehner }}^{2,7 \wedge}$, Norman Wolmark ${ }^{1 \wedge}$, Takayuki Yoshino ${ }^{8 \wedge}$ \\ ${ }^{1}$ NSABP, NRG Oncology, University of Pittsburgh, Pittsburgh, PA, USA; ${ }^{2}$ Helen Diller Family Comprehensive Cancer Center, University of \\ California at San Francisco, San Francisco, CA, USA; ${ }^{3}$ Department of Surgery and Science, Kyushu University, Kyushu, Japan; ${ }^{4}$ Department of \\ Biostatistics and Bioinformatics, Duke University, Durham, NC, USA; ${ }^{5}$ Department of Biostatistics, Precision Oncology, Exact Sciences Corporation, \\ Redwood City, CA, USA; ${ }^{6}$ Global Medical Affairs, Precision Oncology, Exact Sciences Corporation, Redwood City, CA, USA; ${ }^{7}$ Medical Department, \\ Precision Oncology, Exact Sciences Corporation, Redwood City, CA, USA; ${ }^{8}$ National Cancer Center Hospital East, Kashiwa, Japan \\ Contributions: (I) Conception and design: All authors; (II) Administrative support: None; (III) Provision of study materials or patients: None; (IV) \\ Collection and assembly of data: G Yothers, AP Venook, E Oki, D Niedzwiecki, N Wolmark, T Yoshino; (V) Data analysis and interpretation: \\ MR Crager, Y Lin; (VI) Manuscript writing: All authors; (VII) Final approval of manuscript: All authors. \\ Correspondence to: Greg Yothers, PhD. NSABP, NRG Oncology, University of Pittsburgh, Pittsburgh, PA 15213-1516, USA. \\ Email: YothersG@NRGOncology.org.
}

Background: Individualized estimates of the risk of recurrence in colon cancer patients are needed that reflect current medical practice and available treatment options.

Methods: Three validation studies of the 12-gene colon recurrence score assay were used with pre-specified patient-specific meta-analysis (PSMA) methods to integrate the 12-gene Oncotype DX Colon Recurrence Score result (RS) with the clinical and pathology risk factors stage, T-stage, mis-match repair (MMR) status, and number of nodes examined to calculate individualized recurrence risk estimates. Baseline risk estimation used the most recent studies, so the risk estimates reflect current medical practice. The effect of fluorouracil $(5 \mathrm{FU})$ was estimated with a meta-analysis of two studies. The effect of oxaliplatin was estimated using one of the RS assay validation studies, in which patients were randomized to $5 \mathrm{FU}$ with or without oxaliplatin.

Results: The RS result and each of the clinical-pathologic factors provided independent prognostic information for recurrence. Among stage II, T3, MMR-proficient patients with $\geq 12$ nodes examined (the most common scenario), patients with $\mathrm{RS} \leq 30$ (approximately 48\%) have estimated 5 -year recurrence risk $\leq 10 \%$ with surgery alone. Among stage IIIA/B, T3, MMR-deficient patients with $\geq 12$ nodes examined, patients with $\mathrm{RS} \leq 19$ (approximately $14 \%$ ) have an estimated 5 -year recurrence risk $\leq 10 \%$ with surgery alone. Among stage IIIA/B, T3, MMR-proficient patients with $\geq 12$ nodes examined, those with RS $\leq 14$ (approximately 6\%) have estimated 5 -year recurrence risk $\leq 10 \%$ with $5 \mathrm{FU}$ alone.

Discussion: The PSMA integrates the 12-gene colon RS result with clinical and pathology factors to provide individualized recurrence risk estimates that reflect current medical practice. The risk estimates are in a range that may help inform treatment decisions for a substantial number of stage II and stage III patients.

Keywords: Colon cancer; recurrence risk; 12-gene assay; meta-analysis

^ ORCID: Greg Yothers, 0000-0002-7965-7333; Alan P. Venook, 0000-0001-9749-6548; Eiji Oki, 0000-0002-9763-9366; Donna Niedzwiecki, 0000-0002-3566-0450; Yan Lin, 0000-0001-9413-3960; Michael R. Crager, 0000-0002-3594-6299; Calvin Chao, 0000-0002-9704-9227; Frederick L. Baehner, 0000-0001-6883-1212; Norman Wolmark, 0000-0001-7156-1110; Takayuki Yoshino, 0000-0002-0489-4756. 
Submitted Sep 24, 2021. Accepted for publication Jan 04, 2022.

doi: 10.21037/jgo-21-620

View this article at: https://dx.doi.org/10.21037/jgo-21-620

\section{Introduction}

Colorectal cancer is the third most common cancer in the United States, with approximately 145,000 new cases diagnosed annually. Many of these patients present without evidence of metastatic disease where surgical resection of the primary is potentially curative. Most decisions regarding the indications for and specific adjuvant chemotherapy regimens in both stage II and stage III colon cancer are based on recurrence risk assessment using conventional clinical and pathologic risk factors. The 12-gene Oncotype DX Colon Recurrence Score ${ }^{\circledR}$ result (RS) test (Genomic Health, Inc., Redwood City, CA, USA, a wholly owned subsidiary of Exact Sciences) is a validated multigene assay that assesses recurrence risk in stage II and stage III colon cancer patients independent of conventional clinical and pathologic risk factors.

Since the late 1990's the overall rate of recurrence of colon cancer has declined (1). This reflects improvements in surgery, more accurate clinical and pathologic staging and the impact of chemotherapy in selected patients. However, there is no doubt that treatment decisions based on conventional clinical and pathologic lead to the unnecessary use of chemotherapy in some patients and its ineffective use in others. Prognostic tools are still needed to provide more precise, individualized estimates for the risk of recurrence of an individual patient. Theoretically, integrating information from the RS result and clinical-pathologic risk factors such as T-stage, number of lymph nodes examined, mis-match repair (MMR) status, and stage would add more precision, and it would be useful to provide recurrence risk estimates for specific treatment scenarios.

The clinical utility of the RS result is currently limited by reporting recurrence estimates from individual studies only. For stage II colon cancer patients, the report provides 3 -year recurrence risk in patients managed by surgery alone using the QUASAR RS validation study (2). To estimate the effects of adjuvant chemotherapy, physicians must extrapolate by assuming a relative benefit from adjuvant fluorouracil (5FU)-based chemotherapy, approximately $20 \%$ based on the parent QUASAR study. To help physicians understand the effects of adding (or withholding) oxaliplatin in both stage II and III colon cancer patients, the Oncotype
$\mathrm{DX}^{\circledR}$ patient report provides 5 -year risk of recurrence estimates in patients from the National Surgical Adjuvant Breast and Bowel Project (NSABP) C-07 validation study, in which patients were randomized to treatment with $5 \mathrm{FU}$ monotherapy or $5 \mathrm{FU}+$ oxaliplatin.

To improve the accuracy, precision and utility of test results, we calculated recurrence risk using patientspecific meta-analysis (PSMA) for stage II and stage III patients, combining recurrence risk estimates from three clinical validation studies for the RS assay. The metaanalysis integrated the RS result with other prognostic conventional clinical and pathologic risk factors, yielding refined recurrence risk estimates for treatment scenarios of surgery alone, surgery with adjuvant $5 \mathrm{FU}$ chemotherapy, and surgery with $5 \mathrm{FU}$ and oxaliplatin, that more accurately reflect expected outcomes from patients seen today.

We present the following article in accordance with the PRISMA reporting checklist (available at https://jgo. amegroups.com/article/view/10.21037/jgo-21-620/rc).

\section{Methods}

Three RS validation studies were available for the metaanalysis: CALGB 9581, NSABP C-07 and the SUNRISE study. As described in the original reports (3-5), the parent studies were approved by institutional review boards and all study participants provided informed consent. In each of three RS validation studies, tumor grade was centrally assessed according to the College of American Pathologists Consensus Statement.

The parent CALGB 9581 study (3) randomly assigned 1,713 patients with stage II colon cancer to treatment with edrecolomab or observation and found no survival difference. Venook et al. (6) reported a prospectiveretrospective study of the association of the RS result with recurrence using a stratified cohort sample consisting of all 162 patients, pooling patients randomized to observation and edrecolomab, with available tissue and recurrence and a random selection of nonrecurring patients stratified on treatment for a total of 690 patients. CALGB 9581 enrolled from 1997 through 2002.

The NSABP C-07 study (4) (enrollment 2000-2002) randomly assigned patients with stage II and III colon 
cancer to $5 \mathrm{FU}$ or $5 \mathrm{FU}$ plus oxaliplatin. Yothers et al. (7) reported a prospective-retrospective study of the association of the RS result with recurrence in 892 randomly selected patients from this study (half the patients with available tissue), 245 of whom experienced recurrence.

The SUNRISE Study in Japan reported by Yamanaka et al. (5) included a stratified cohort sample from 1,487 consecutive patients from 2000 through 2005 with stage II or III colon cancer who had surgery alone. The sample included 630 patients (1:2 ratio of recurrence to nonrecurrence). Sampling was stratified by stage (II $v s$. III), 597 of whom were evaluable for analysis with 202 experiencing recurrence.

\section{Statistical analysis}

The statistical methods were pre-specified and approved in advance by the investigators. Cox proportional hazards models were fit separately for each study with effects for RS result, number of nodes examined $(<12$ or $\geq 12)$, T-stage (T4 or T1-3), MMR status (proficient/unknown or deficient) and, where applicable, stage (II, IIIA/B or IIIC) and treatment (oxaliplatin $+5 \mathrm{FU}$ or $5 \mathrm{FU}$ ). The endpoint was recurrence-free interval, defined as time to recurrence or death due to colon cancer. Patients who died without recurrence were censored at last follow-up. Since CALGB 9581 and the SUNRISE study used stratified cohort sampling (8), they were analyzed using the inverse stratum-specific sampling ratio as a weight with the robust regression parameter variance estimator of Lin and Wei (9). The significance of each factor in the multivariate models adjusting for the other factors was tested using a metaanalysis Wald test since likelihood ratio tests are not valid for cohort-sampling studies. The risk of recurrence at 1, 3 and 5 years after surgery was estimated using the "special population" PSMA method (10) to adjust for the effect of stage and oxaliplatin treatment. The log hazard ratio (HR) for the effect of $5 \mathrm{FU}$ was estimated separately for stage II and III using a fixed effects meta-analysis of $\log$ HR's extracted from reports of the QUASAR study (11) and the pooled analysis of NSABP trials reported by Wilkinson and colleagues (12) and integrated into the PSMA risk estimation. Since the overall recurrence risk has decreased over time, the events from the latest-enrolling two studies were used to estimate the baseline cumulative hazard. Potential interaction among the model factors was assessed using meta-analysis Wald tests weighting individual study interaction effect estimates by their inverse variance. Details of the statistical methods are available online: https://cdn. amegroups.cn/static/public/jgo-21-620-1.pdf, including documentation of the originally planned methods. The only change from the planned methods was to estimate the effect of $5 \mathrm{FU}$ separately by stage (II $v s$. III) rather than overall. Analyses were conducted using SAS ${ }^{\circledR}$ Version 9.2 and SAS/Stat Version 14.1 (SAS Institute, Cary, NC, USA).

\section{Results}

Patient and tumor characteristics in the enrolled study populations are shown in Table 1 and the distribution of the RS result integrated across the three studies is shown in Figure S1. Overall, $17 \%$ of patients had RS $\leq 20,31 \%$ had $\mathrm{RS} \leq 25,48 \%$ had $\mathrm{RS} \leq 30$, and $22 \%$ had RS $>40$; the median RS result was 30 [interquartile range (IQR), 23-39] with mean 32 and standard deviation 12 . There were relatively few T4 tumors in CALGB 9581 and C-07. The Cox model covariates were largely uncorrelated, with a maximum absolute correlation of only 0.13 (Table S1).

The Cox proportional hazards regression model fit to each of the three studies is shown in Table 2. The direction of association of each of the covariates with recurrence risk is consistent across studies. The HR's vary across studies, but meta-analysis Wald tests indicated that each covariate provides significant prognostic information independent of all the others. Meta-analysis Wald tests indicated no significant interaction among the common covariates RS result, number of nodes examined ( $<12$ or $\geq 12)$, T-stage and MMR deficiency or between each of the common covariates and stage or treatment with oxaliplatin (Figures S2-S4). Therefore, the main effects model was judged appropriate. Exploratory meta-analyses examining tumor grade and right-sided tumor location showed no significant prognostic value for recurrence of either of these in either univariable or multivariable analysis together with the pre-planned covariates stage, RS result, number of nodes examined, T-stage and MMR deficiency (Tables S2,S3, Figure S5).

The HR's for $5 \mathrm{FU}$ for recurrence-free interval, based on a meta-analysis of two large studies $(11,12)$, are shown in Figure 1. The estimated effect of $5 \mathrm{FU}$ in reducing the recurrence rate is larger in stage III $[\mathrm{HR}=0.63 ; 95 \%$ confidence interval (CI): 0.54-0.73] than in stage II (HR $=0.77 ; 95 \%$ CI: $0.66-0.91$ ). This difference was observed consistently across the two studies with a meta-analysis stage-by-treatment interaction test $\mathrm{P}$ value of 0.075 .

A meta-analysis estimate of the absolute standardized 
Table 1 Patient and tumor characteristics

\begin{tabular}{|c|c|c|c|}
\hline Characteristic & CALGB 9581*\# $(n=690)$ & SUNRISE $^{*}(n=597)$ & NSABP C-07 $(n=892)$ \\
\hline \multicolumn{4}{|l|}{ Number of patients (events) } \\
\hline Surgery alone & 690 [162] & 597 [202] & - \\
\hline Surgery + 5FU & - & - & 449 [135] \\
\hline Female gender & $330(48 \%)$ & $278(47 \%)$ & $379(42 \%)$ \\
\hline \multicolumn{4}{|l|}{ Age } \\
\hline Median (IQR) & $66(57-73)$ & $66(57-72)$ & $60(51-67)$ \\
\hline Min-max & $30-90$ & $23-80$ & $22-85$ \\
\hline MMR deficient & $137(20 \%)$ & $30(5 \%)$ & $84(9 \%)$ \\
\hline MMR proficient & 519 (75\%) & 567 (95\%) & $626(70 \%)$ \\
\hline MMR status unknown & $34(5 \%)$ & $0(0 \%)$ & $182(20 \%)$ \\
\hline \multicolumn{4}{|l|}{ RS result } \\
\hline Median (IQR) & $31(25-39)$ & $28(20-37)$ & $33(25-41)$ \\
\hline Min-max & $2-78$ & $0-68$ & $4-80$ \\
\hline Stage II & $690(100 \%)$ & 247 (69\%) & $264(30 \%)$ \\
\hline Stage IIIA/B & - & $278(25 \%)$ & $409(46 \%)$ \\
\hline Ascending colon & $133(20 \%)$ & $109(20 \%)$ & $160(18 \%)$ \\
\hline Transverse colon ${ }^{+}$ & $159(23 \%)$ & $68(10 \%)$ & $202(23 \%)$ \\
\hline Descending colon & $42(6 \%)$ & $36(6 \%)$ & $50(6 \%)$ \\
\hline Sigmoid colon/rectosigmoid & $182(26 \%)$ & $336(55 \%)$ & $311(35 \%)$ \\
\hline Right-sided tumor location^^ & $423(62 \%)$ & 225 (38\%) & 489 (55\%) \\
\hline
\end{tabular}

*, percentage and summary statistics calculated using stratified cohort sampling weights; ", includes patients randomized to edrecolomab in CALGB 9581. Edrecolomab was ineffective; ${ }^{+}$, includes hepatic flexure, splenic colon and splenic flexure; $\wedge$, defined as the cecum, ascending colon, hepatic flexure and transverse colon. NSABP, National Surgical Adjuvant Breast and Bowel Project; 5FU, fluorouracil; IQR, interquartile range; MMR, mis-match repair; RS, 12-gene Oncotype DX Colon Recurrence Score result.

HR (13) for the joint contributions of all the covariates, including stage, to separation of risk in the population, controlling for treatment, is 2.19 (95\% CI: 1.69-2.84), meta-analysis Wald test $\mathrm{P}<0.001$. This meta-analysis estimate weighted the partial absolute standardized log HR from each study by its effective sample size, accounting 
Table 2 Cox proportional hazards regression model HR with $95 \%$ CI and Wald test $\mathrm{P}$ values

\begin{tabular}{|c|c|c|c|c|}
\hline Effect & $\begin{array}{c}\text { CALGB } 9581(n=690)^{a} \\
162 \text { events }\end{array}$ & $\begin{array}{l}\text { SUNRISE }(n=597)^{a}, \\
202 \text { events }\end{array}$ & $\begin{array}{c}\text { NSABP C-07 }(n=892) \\
245 \text { events }\end{array}$ & Meta-analysis Wald test \\
\hline RS, per 25 units & $1.57(1.14,2.18) ; P=0.006$ & 1.95 (1.35, 2.82); $P<0.001$ & 1.80 (1.39, 2.34); $P<0.001$ & $1.76(1.47,2.11) ; P<0.001$ \\
\hline T4 vs. T1-3 & $1.04(0.51,2.13) ; P=0.91$ & $1.19(0.81,1.75) ; P=0.38$ & 2.71 (1.81, 4.06); $P<0.001$ & $1.64(1.27,2.13) ; \mathrm{P}<0.001$ \\
\hline $\begin{array}{l}\text { MMR deficient vs. proficient/ } \\
\text { unknown }\end{array}$ & $0.62(0.39,1.00) ; P=0.051$ & $0.70(0.30,1.60) ; P=0.39$ & $0.29(0.13,0.65) ; P=0.003$ & $0.54(0.38,0.79) ; P=0.001$ \\
\hline Stage IIIA/B vs. II & - & 2.29 (1.62, 3.23); $P<0.001$ & $2.32(1.55,3.46) ; P<0.001$ & 2.30 (1.77, 2.99); $\mathrm{P}<0.001$ \\
\hline Stage IIIC vs. II & - & 5.30 (3.41, 8.24); $\mathrm{P}<0.001$ & 5.53 (3.69, 8.29); $P<0.001$ & $5.42(4.02,7.31) ; P<0.001$ \\
\hline Oxali + 5FU vs. 5FU & - & - & $0.80(0.62,1.03) ; P=0.089$ & - \\
\hline
\end{tabular}

a , study used stratified cohort sampling. HR, hazard ratios; Cl, confidence intervals; NSABP, National Surgical Adjuvant Breast and Bowel Project; RS, 12-gene Oncotype DX Colon Recurrence Score result; MMR, mis-match repair; Oxali, oxaliplatin; 5FU, fluorouracil.

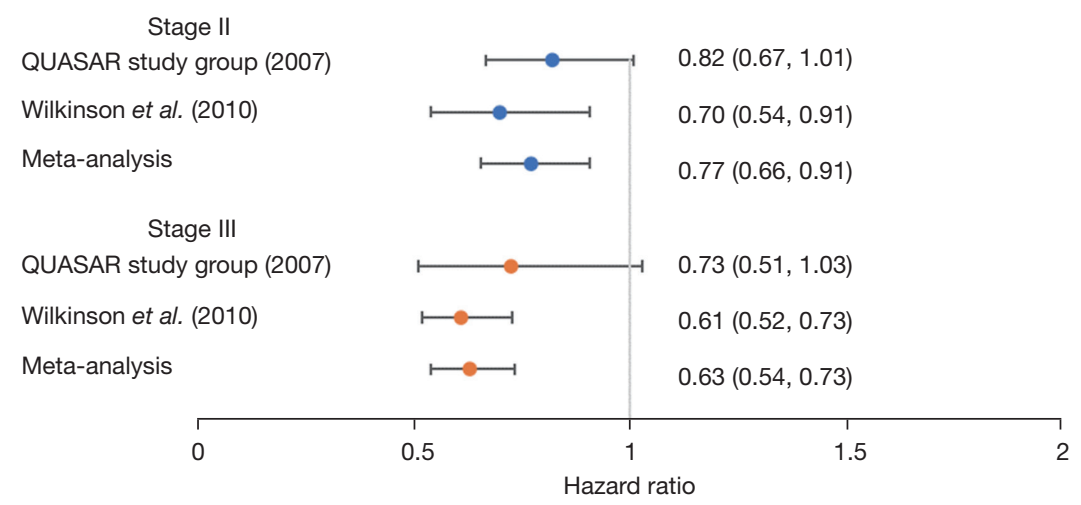

Figure 1 Meta-analysis estimate of $5 \mathrm{FU}$ treatment effect HR by stage. Meta-analysis test for interaction of stage with treatment $\mathrm{P}=0.075$. $5 \mathrm{FU}$, fluorouracil; HR, hazard ratio.

for stratified cohort sampling. A similar meta-analysis estimate of the partial absolute standardized HR (14) for the combination of the four covariates RS result, number of nodes examined ( $<12 v s . \geq 12)$, MMR-deficiency and T-stage, controlling for stage (II, IIIA/B or IIIC) and treatment, is 1.55 (95\% CI: 1.29-1.86), meta-analysis Wald test $\mathrm{P}<0.001$. As shown in Figure 2, RS result contributed an estimated $48 \%$ [standard error (SE) $15 \%$ ] of the risk score variance, MMR deficiency 30\% (SE 14\%), number of nodes examined 17\% (SE 9\%) and T-stage 5\% (SE 3\%). Percentage contribution is a measure of variable's prognostic importance for recurrence in the studied population.

In view of an analysis of pooled data from MMRdeficient patients in several randomized trials of $5 \mathrm{FU}$ by Sargent and colleagues (15), which indicated that $5 \mathrm{FU}$ alone is not effective, particularly in stage II patients, we decided not to provide recurrence risk estimates with $5 \mathrm{FU}$ treatment for MMR-deficient patients. Cohen and colleagues (16) also concluded that fluoropyrimidine (FP) is ineffective compared to surgery alone in MMR-deficient patients.

Example estimates and $95 \%$ CI for the 5-year recurrence risk for scenarios based on number of nodes examined and MMR status are shown in Figure 3 for stage II and IIIA/B, T3 patients. The complete set of 5-, 3- and 1-year recurrence risk estimates are available online: https:// cdn.amegroups.cn/static/public/jgo-21-620-1.pdf. The various curves show the impact of each of the factors on the recurrence risk. As indicated by the lack of correlation among RS and the other covariates in the analysis, the distribution of RS is essentially unchanged across the different covariate levels. For stage II, T3, MMR-proficient patients with $\geq 12$ nodes examined (the most common 


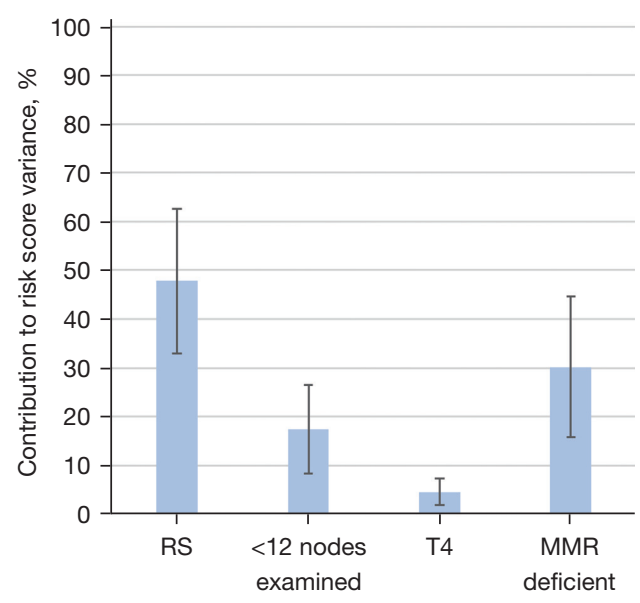

Figure 2 Meta-analysis estimate (with SE) of proportional contributions of each of the common covariates to the risk score variance, controlling for stage and treatment effects. RS, 12-gene Oncotype DX Colon Recurrence Score result; MMR, mis-match repair; SE, standard error.

scenario), patients with RS $\leq 30$ (approximately $48 \%$ of patients) have an estimated 5 -year recurrence risk $\leq 10 \%$ with surgery alone. Among stage IIIA/B, T3, MMRdeficient patients with $\geq 12$ nodes examined, patients with RS $\leq 19$ (approximately 14\%) have an estimated 5-year recurrence risk $\leq 10 \%$ with surgery alone. Among stage IIIA/B, T3, MMR-proficient patients with $\geq 12$ nodes examined, those with RS $\leq 14$ (approximately $6 \%$ ) will have estimated 5 -year recurrence risk $\leq 10 \%$ with $5 \mathrm{FU}$ alone.

Comparisons of recurrence risk estimates from the PSMA with corresponding risk estimates from the existing Oncotype DX Colon Recurrence Score patient report are shown in Figure 4. The existing report provides 3-year risk estimates for stage II, MMR-proficient patients receiving surgery alone that depend on T-stage and RS result. For stage III patients, the existing report provides 5 -year recurrence risk estimates for 5FU alone and 5FU with oxaliplatin, pooling across T-stages. The recurrence risk estimates provided by the PSMA are generally lower, although some for stage III patients are higher since the PSMA accounts for T-stage and number of nodes examined.

\section{Discussion}

In this analysis, the RS result and conventional clinical and pathologic risk factors were integrated using PSMA. These known risk factors for recurrence have been reported previously $(5-7,17)$. The meta-analysis showed that the RS result and the conventional clinical and pathologic risk factors T-stage, number of nodes examined, AJCC stage, and MMR status are largely independent of each other and furthermore are independently associated with the risk of recurrence. Treatment effects were estimated using a meta-analysis of two studies of $5 \mathrm{FU}$ and one of the three RS validation studies in which patients were randomized to $5 \mathrm{FU}$ alone or $5 \mathrm{FU}$ plus oxaliplatin. The integrated RS result and conventional risk factors provided refined recurrence risk estimates for untreated and 5FU- and oxaliplatin-treated stage II and stage III patients. These results more accurately reflect expected outcomes from patients seen today.

Cohen and colleagues (16) performed a pooled analysis of randomized studies of FP with oxaliplatin $v s$. FP alone in stage III colon cancer, which found an HR for diseasefree survival of 0.47 (95\% CI: $0.27-0.82)$ in microsatellite instability (MSI)/MMR-deficient patients and 0.82 (95\% CI: $0.70-0.97$ ) in microsatellite stable (MSS)/MMR-proficient patients. The HR of 0.47 in MMR-deficient patients is similar to the combined effects in stage III patients of $5 \mathrm{FU}$ and oxaliplatin $\left(\mathrm{HR}_{5 \mathrm{FU}} \times \mathrm{HR}_{\text {oxali }}=0.63 \times 0.80=0.50\right)$ from the present analysis, suggesting that the addition of oxaliplatin may allow MMR-deficient patients to also benefit from 5FU. The HR 0.82 in MMR-proficient patients is similar to the effect of oxaliplatin with $5 \mathrm{FU} v s$. $5 \mathrm{FU}$ alone (0.80) in the present analysis, which would be expected if $5 \mathrm{FU}$ alone were effective in these patients (isolating the effect of oxaliplatin). These corroborating results suggest that the risk estimates for $5 \mathrm{FU}$ + oxaliplatin treatment in patients with stage III MMR-deficient tumors provided by the present analysis are appropriate.

The RS result is calculated from an analytically validated and standardized quantitative reverse transcriptase polymerase chain reaction assay that measures the expression of 12 genes (seven recurrence genes and five reference genes) in formalin-fixed paraffin-embedded (FFPE) primary colon tumor tissue (18). The applicability of the RS result is reinforced by the identity of the recurrence genes and the measured changes in tumor biology produced by their dysregulation. Six of the seven genes are in two key biologic pathways: cell cycle control (MKI67, MYC, MYBL2) and stromal response (FAP, BGN, $I N H B A)$. The seventh recurrence gene $(G A D D 45 B)$ is a marker of genotoxic stress and may regulate activity of stromal response genes, including $B G N(18)$.

Since the RS result contributes prognostic information 

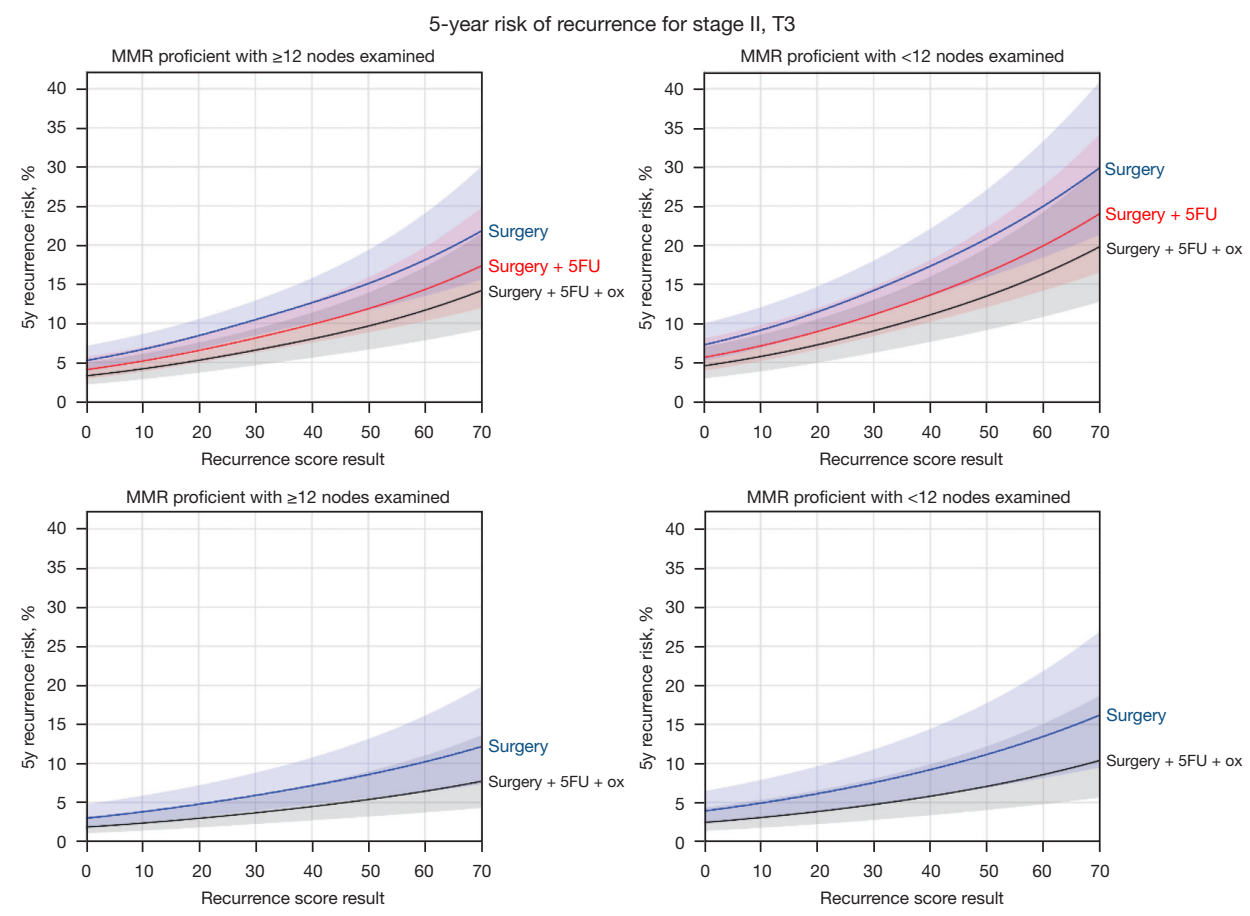

5-year risk of recurrence for stage IIIAB, T1-T3
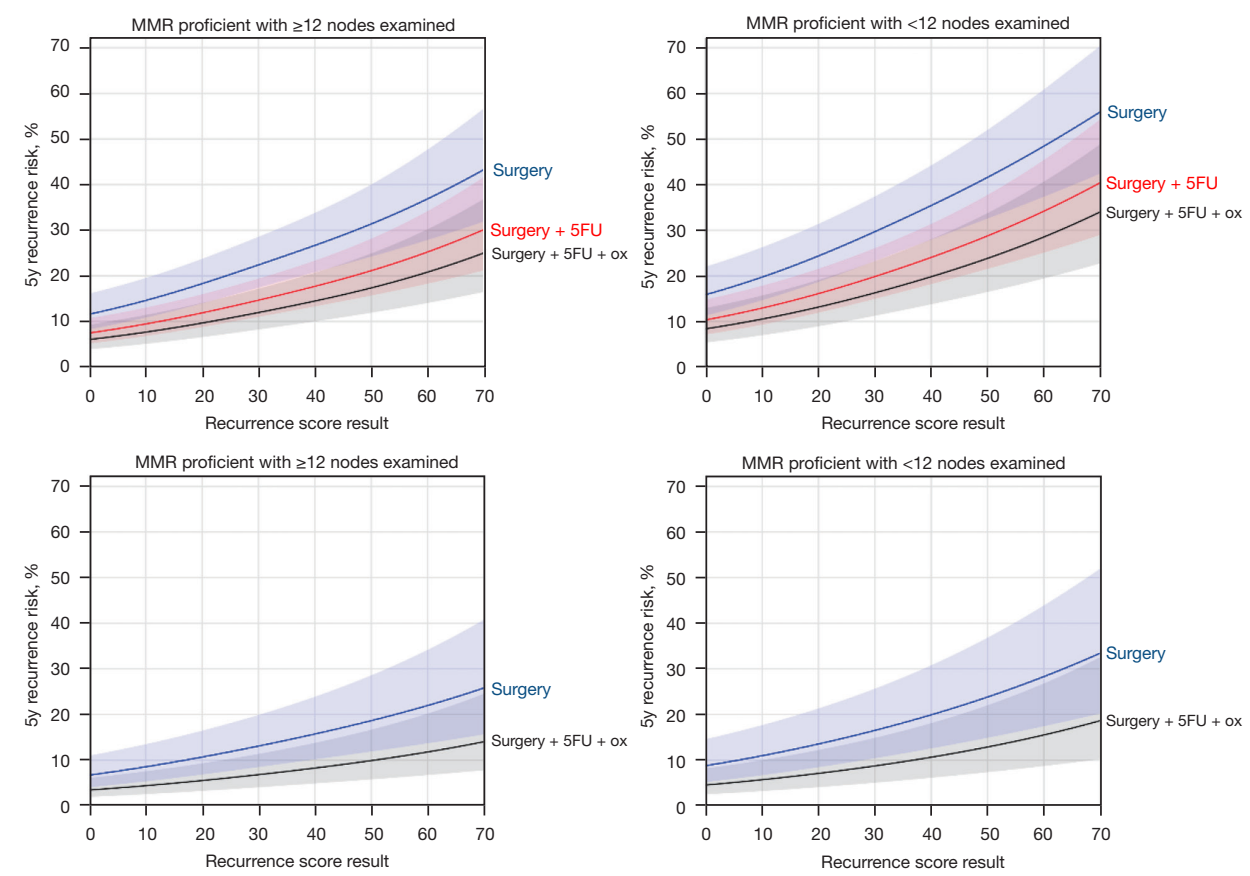

Figure 3 Example PSMA 5-year recurrence risk estimates and 95\% CI. 5FU, fluorouracil; MMR, mis-match repair; PSMA, patient-specific meta-analysis; CI, confidence intervals. 

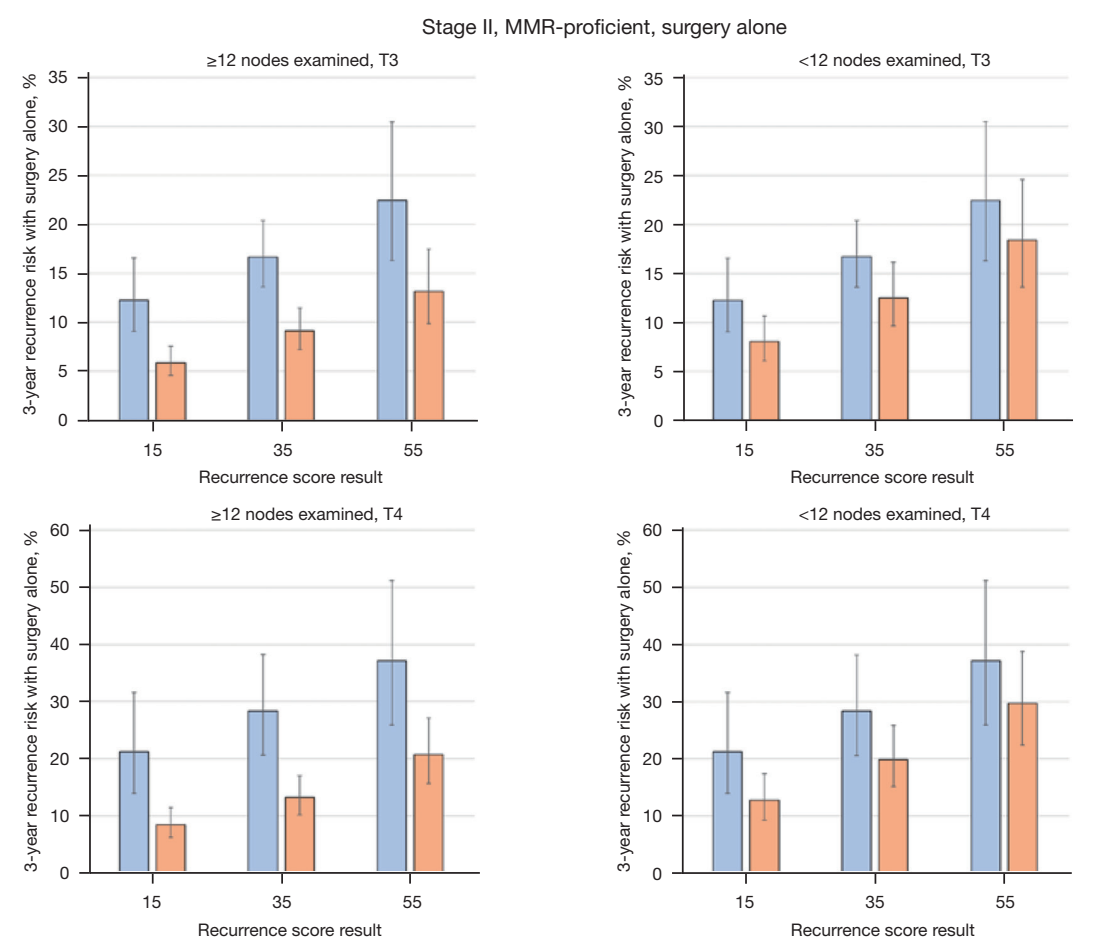

Stage IIIA/B, MMR-proficient, surgery with 5FU
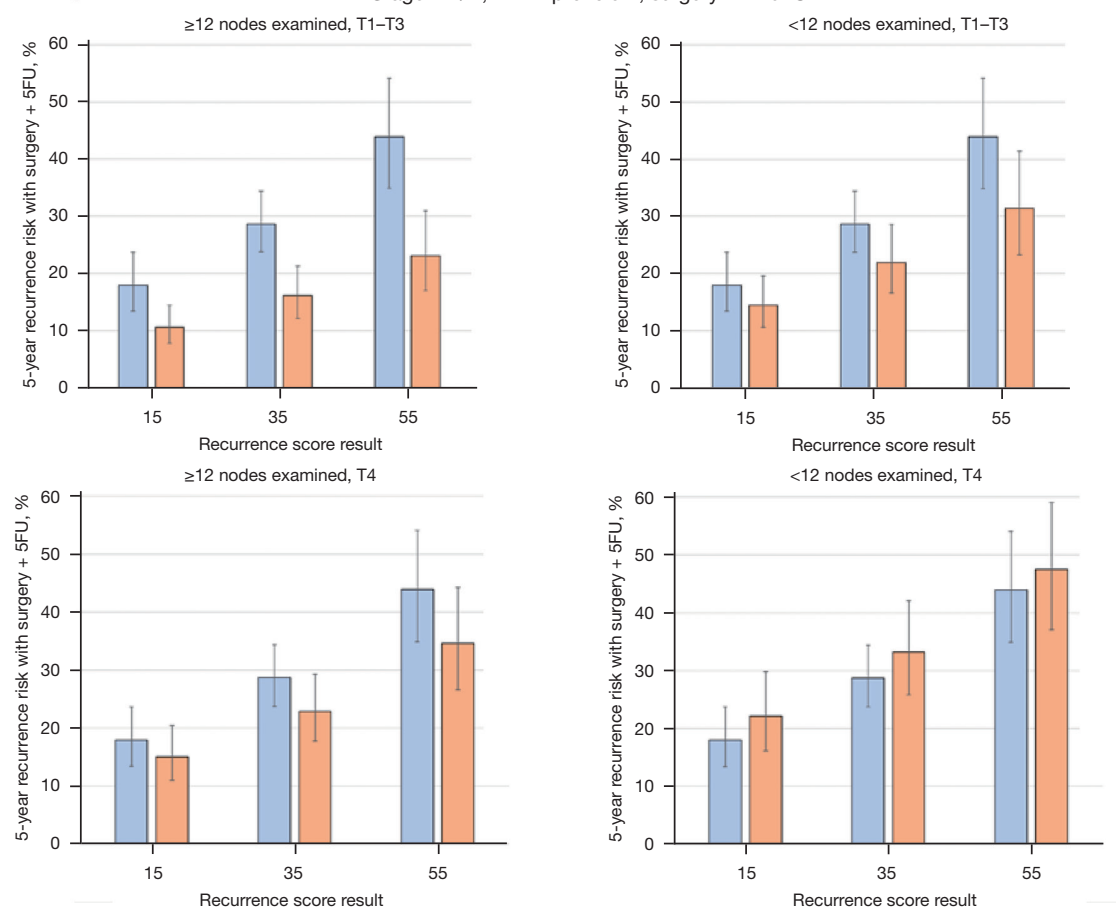

Figure 4 Comparison of recurrence risk estimates (95\% CI) from the PSMA and the existing Oncotype DX Colon Recurrence Score patient report (Genomic Health, Inc., a wholly owned subsidiary of Exact Sciences Corporation). MMR, mis-match repair; PSMA, patientspecific meta-analysis; 5FU, fluorouracil; CI, confidence intervals. 
independent of the conventional clinical and pathologic tumor features, the combination provides more prognostic information than the conventional clinical and pathologic features alone. RS result contributes about half of the total risk score variance, with the rest coming from the combined contributions of the conventional factors. The proportional contribution of each variable is influenced by its strength of association with the risk of recurrence and its distribution in the population. For example, the contribution of T-stage is small because relatively few tumors are $\mathrm{T} 4$ and since its association with recurrence is modest. Relatively few tumors are MMR-deficient but the association of MMR-deficiency with a reduction in recurrence risk is strong, so MMR contributes substantially more than T-stage to the risk score variance.

Strengths of this meta-analysis include a large data set, which allows more individualized risk estimates while maintaining reasonable precision (CI width), and the availability of risk estimates for various treatment scenarios. Limitations include that the estimated effect of $5 \mathrm{FU}$ is from a meta-analysis of a randomized study (11) and a non-randomized treatment comparison with covariate adjustment to reduce bias (12). However, the consistency of the results between these two studies suggests that the nonrandomized comparison is providing reliable results. The SUNRISE study was a retrospective analysis that selected patients who had not received adjuvant chemotherapy after resection for stage II or III colon cancer and this may have led to selection of patients whom clinicians had considered to be at lower risk of recurrence, although patients in this study were treated during a time period when adjuvant chemotherapy for stage III disease was not used in Japan. Also, the PSMA risk assessment used a baseline risk assessment from the last two enrolling studies (NSABP C-07, enrolling from 2000-2002 and SUNRISE, enrolling from 2000-2005). If further improvements in patient outcomes have occurred since this time, they are not reflected in the present recurrence risk estimates. Finally, the RS result is not predictive, that is, it is not associated with the relative treatment effect of chemotherapy with $5 \mathrm{FU}$ or oxaliplatin. Therefore, the differences in absolute risk reduction due to chemotherapy with $5 \mathrm{FU}$ potentially with oxaliplatin reflect the recurrence risk together with a relative chemotherapy effect that is constant over the range of RS results. Nevertheless, having the risk estimates sideby-side for each treatment scenario should help facilitate discussions between physician and patient of the risks and benefits of each treatment option. The PSMA assesses these risk and benefits in a patient-specific way, helping to personalize the decision-making process.

Part of the difference in recurrence rates between the existing patient report for stage II patients, which is based on the QUASAR RS validation study (2), and the current PSMA is due to a larger number of patients with $<12$ nodes examined (63\%) in the QUASAR study. However, additional factors appear to be at play since risk estimates adjusted for number of nodes examined, T-stage, MMR-status and RS result still show decreases over time. Improved surgical techniques, better imaging, and other changes in the standard of medical care may have contributed to the decrease in risk since the 1990s. Regardless of the cause, it is important that recurrence risk estimates reflect the current standard of care.

The PSMA recurrence risks should be useful for treatment decisions for many patients. Five-year recurrence risk estimates with surgery alone for patients with stage II, T3, MMR-proficient tumors and $\geq 12$ nodes examined are $\leq 10 \%$ for RS $\leq 30$ (about $48 \%$ of patients). Similarly, for patients with stage IIIA/B, T3, MMR-deficient tumors with $\geq 12$ nodes examined, patients with $\mathrm{RS} \leq 19$ (approximately $14 \%$ ) have 5 -year recurrence risk $\leq 10 \%$ with surgery alone. The low recurrence risk for these groups of patients could assist in considerations of whether to omit chemotherapy. Among stage IIIA/B, T3, MMR-proficient patients with $\geq 12$ nodes examined, those with $\mathrm{RS} \leq 14$ (approximately $6 \%$ ) have estimated 5 -year recurrence risk $\leq 10 \%$ with $5 \mathrm{FU}$ alone, which could inform the considerations of omitting oxaliplatin. The risk estimates for patients with stage II MMR-deficient tumors are uniformly low, regardless of RS result and the clinical-pathology factors, so the RS result is not considered clinically useful for these patients. The oneyear recurrence risk estimates may be useful in identifying patients at exceptionally high risk for an early recurrence who might benefit from additional adjuvant intervention.

In summary, the PSMA integrates the RS result with clinical and pathology factors to provide individualized recurrence risk estimates that reflect current medical practice. The risk estimates are in a range that may inform treatment decisions for a substantial number of stage II and stage III patients. Since even the most recent studies used in this meta-analysis enrolled about 15-20 years ago, it would be useful to validate the risk estimates in a current, independent cohort of patients to ensure that the observed association of recurrence risk with the gene expression and other the factors is still accurate. Future work could consider other gene sets identified using next generation 
sequencing (NGS) and should evaluate the combination of genomic, clinical and pathology factors with ongoing assessments of circulating tumor DNA to further enhance recurrence rick assessment.

\section{Acknowledgments}

This analysis was presented as a poster at the 2021 ASCO Annual Meeting.

Funding: Funding in support of manuscript preparation was provided by the National Cancer Institute grants \#U10CA180868 and U10CA180822.

\section{Footnote}

Reporting Checklist: The authors have completed the PRISMA reporting checklist. Available at https://jgo. amegroups.com/article/view/10.21037/jgo-21-620/rc

Conflicts of Interest: All authors have completed the ICMJE uniform disclosure form (available at https://jgo.amegroups. com/article/view/10.21037/jgo-21-620/coif). MRC, FLB and $\mathrm{CC}$ are or were employees of and received remuneration and stock from Genomic Health, Inc., a wholly owned subsidiary of Exact Sciences Corporation, which provides test results based on the 12 -gene assay. EO received lecture fees from Bayer Japan, Chugai Pharmaceutical Co., Ltd., Taiho Pharmaceutical Co., Ltd., Eli Lilly, Ono Pharmaceutical Co., Ltd., and Takeda Pharmaceutical Co., Ltd. NW reports support for the present manuscript via NCI grant \#U10CA180868, directly to his institution only. TY reports grants from Taiho Pharmaceutical Co., Ltd., Sumitomo Dainippon Pharma Co., Ltd., Ono Pharmaceutical Co., Ltd., Chugai Pharmaceutical Co., Ltd., Amgen K.K., Parexel International Inc., MSD K.K., Daiichi Sankyo Co., Ltd., and Sanofi K.K., outside the submitted work. The other authors have no conflicts of interest to declare.

Ethical Statement: The authors are accountable for all aspects of the work in ensuring that questions related to the accuracy or integrity of any part of the work are appropriately investigated and resolved.

Open Access Statement: This is an Open Access article distributed in accordance with the Creative Commons Attribution-NonCommercial-NoDerivs 4.0 International License (CC BY-NC-ND 4.0), which permits the non- commercial replication and distribution of the article with the strict proviso that no changes or edits are made and the original work is properly cited (including links to both the formal publication through the relevant DOI and the license). See: https://creativecommons.org/licenses/by-nc-nd/4.0/.

\section{References}

1. Siegel RL, Miller KD, Jemal A. Cancer statistics, 2020. CA Cancer J Clin 2020;70:7-30.

2. Gray RG, Quirke P, Handley K, et al. Validation study of a quantitative multigene reverse transcriptase-polymerase chain reaction assay for assessment of recurrence risk in patients with stage II colon cancer. J Clin Oncol 2011;29:4611-9.

3. Niedzwiecki D, Bertagnolli MM, Warren RS, et al. Documenting the natural history of patients with resected stage II adenocarcinoma of the colon after random assignment to adjuvant treatment with edrecolomab or observation: results from CALGB 9581. J Clin Oncol 2011;29:3146-52.

4. Kuebler JP, Wieand HS, O'Connell MJ, et al. Oxaliplatin combined with weekly bolus fluorouracil and leucovorin as surgical adjuvant chemotherapy for stage II and III colon cancer: results from NSABP C-07. J Clin Oncol 2007;25:2198-204.

5. Yamanaka T, Oki E, Yamazaki K, et al. 12-Gene Recurrence Score Assay Stratifies the Recurrence Risk in Stage II/III Colon Cancer With Surgery Alone: The SUNRISE Study. J Clin Oncol 2016;34:2906-13.

6. Venook AP, Niedzwiecki D, Lopatin M, et al. Biologic determinants of tumor recurrence in stage II colon cancer: validation study of the 12-gene recurrence score in cancer and leukemia group B (CALGB) 9581. J Clin Oncol 2013;31:1775-81.

7. Yothers G, O'Connell MJ, Lee M, et al. Validation of the 12-gene colon cancer recurrence score in NSABP C-07 as a predictor of recurrence in patients with stage II and III colon cancer treated with fluorouracil and leucovorin (FU/LV) and FU/LV plus oxaliplatin. J Clin Oncol 2013;31:4512-9.

8. Gray RJ. Weighted analyses for cohort sampling designs. Lifetime Data Anal 2009;15:24-40.

9. Lin DY, Wei LJ. The robust inference for the Cox proportional hazards model. J Am Stat Assoc 1989;84:1074-8.

10. Crager MR, Tang G. Patient-specific meta-analysis for risk assessment using multivariate proportional hazards 
regression. J Appl Stat 2014;41:2676-95.

11. Quasar Collaborative Group; Gray R, Barnwell J, et al. Adjuvant chemotherapy versus observation in patients with colorectal cancer: a randomised study. Lancet 2007;370:2020-9.

12. Wilkinson NW, Yothers G, Lopa S, et al. Long-term survival results of surgery alone versus surgery plus 5-fluorouracil and leucovorin for stage II and stage III colon cancer: pooled analysis of NSABP C-01 through C-05. A baseline from which to compare modern adjuvant trials. Ann Surg Oncol 2010;17:959-66.

13. Crager MR. Generalizing the standardized hazard ratio to multivariate proportional hazards regression, with an application to clinical genomic studies. J Appl Stat 2012;39:399-417.

14. Crager MR. Extensions of the absolute standardized hazard ratio and connections with measures of explained variation and variable importance. Lifetime Data Anal 2020;26:872-92.

Cite this article as: Yothers G, Venook AP, Oki E, Niedzwiecki D, Lin Y, Crager MR, Chao C, Baehner FL, Wolmark N, Yoshino T. Patient-specific meta-analysis of 12gene colon cancer recurrence score validation studies for recurrence risk assessment after surgery with or without $5 \mathrm{FU}$ and oxaliplatin. J Gastrointest Oncol 2022;13(1):126-136. doi: 10.21037/jgo-21-620
15. Sargent DJ, Marsoni S, Monges G, et al. Defective mismatch repair as a predictive marker for lack of efficacy of fluorouracil-based adjuvant therapy in colon cancer. J Clin Oncol 2010;28:3219-26.

16. Cohen R, Taieb J, Fiskum J, et al. Microsatellite Instability in Patients With Stage III Colon Cancer Receiving Fluoropyrimidine With or Without Oxaliplatin: An ACCENT Pooled Analysis of 12 Adjuvant Trials. J Clin Oncol 2021;39:642-51.

17. O'Connell MJ, Lavery I, Yothers G, et al. Relationship between tumor gene expression and recurrence in four independent studies of patients with stage II/III colon cancer treated with surgery alone or surgery plus adjuvant fluorouracil plus leucovorin. J Clin Oncol 2010;28:3937-44.

18. Clark-Langone KM, Sangli C, Krishnakumar J, et al. Translating tumor biology into personalized treatment planning: analytical performance characteristics of the Oncotype DX Colon Cancer Assay. BMC Cancer 2010;10:691. 


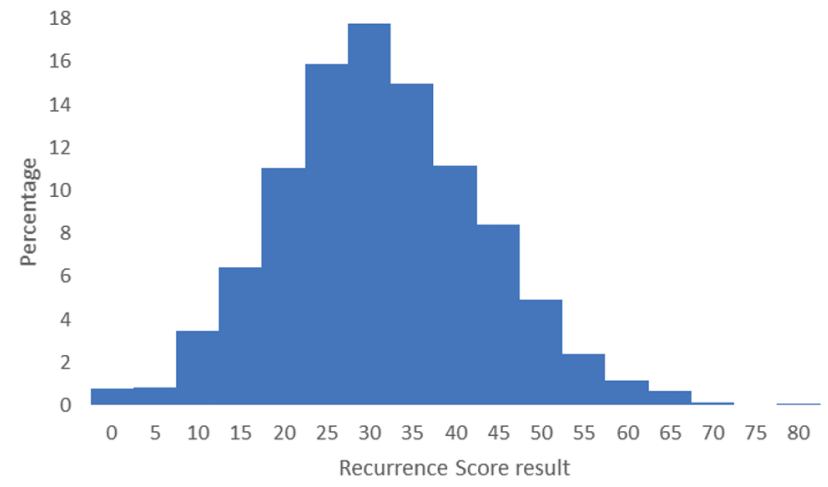

\begin{tabular}{cr}
\hline RS range & \% patients \\
\hline$\leq 20$ & $16.6 \%$ \\
$\leq 25$ & $31.1 \%$ \\
$\leq 30$ & $48.2 \%$ \\
$>40$ & $22.1 \%$
\end{tabular}

Figure S1 Distribution of recurrence score result in meta-analysis population. RS, 12-gene Oncotype DX Colon Recurrence Score result.

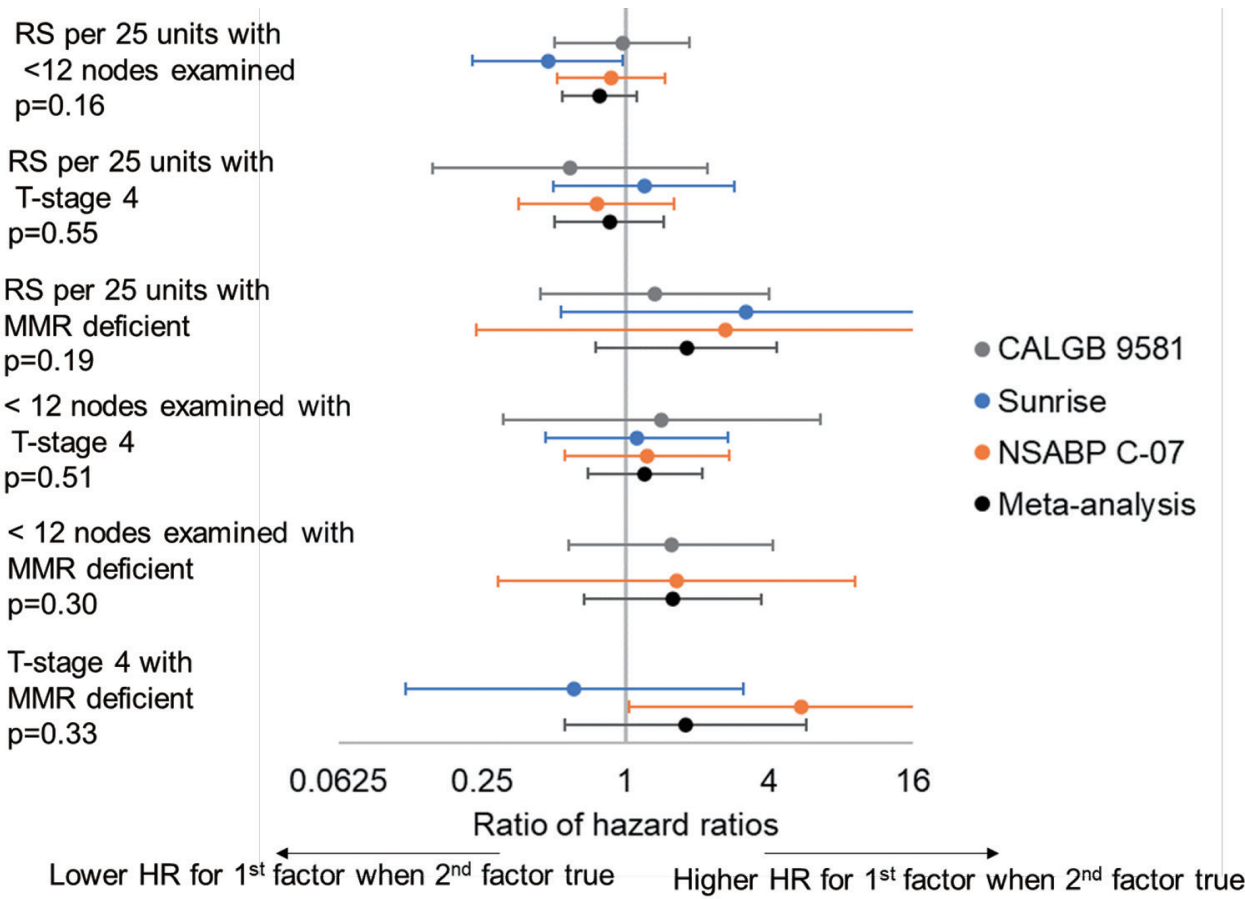

Figure S2 Meta-analysis tests of interaction among common covariates. Ratio of HR (estimates and 95\% CI) with meta-analysis Wald test $\mathrm{P}$ values. Studies with no recurrence event in an interaction category excluded. RS, 12-gene Oncotype DX Colon Recurrence Score result; MMR, mis-match repair; HR, hazard ratios; CI, confidence intervals. 


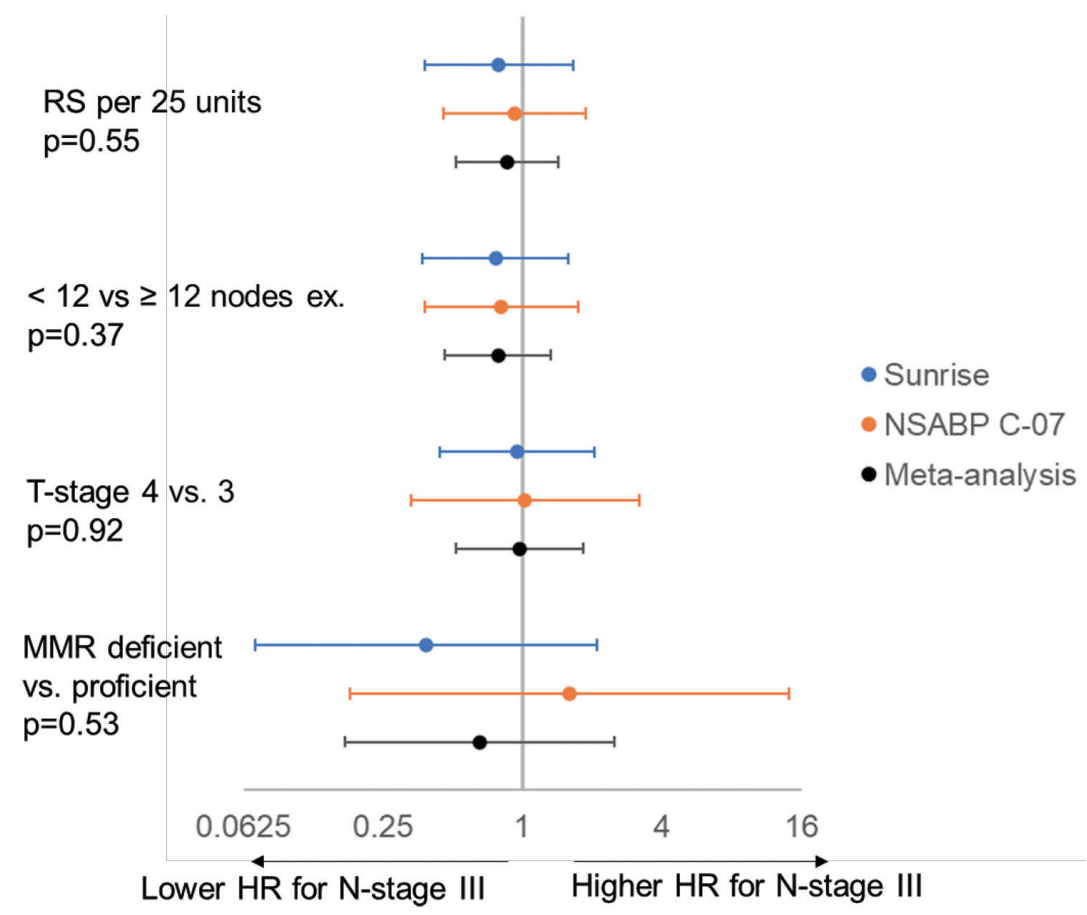

Figure S3 Meta-analysis tests of interaction of common covariates with stage III vs. II. Ratio of HR (estimates and 95\% CI) with meta-analysis Wald test P values. RS, 12-gene Oncotype DX Colon Recurrence Score result; MMR, mis-match repair; HR, hazard ratios; CI, confidence intervals.

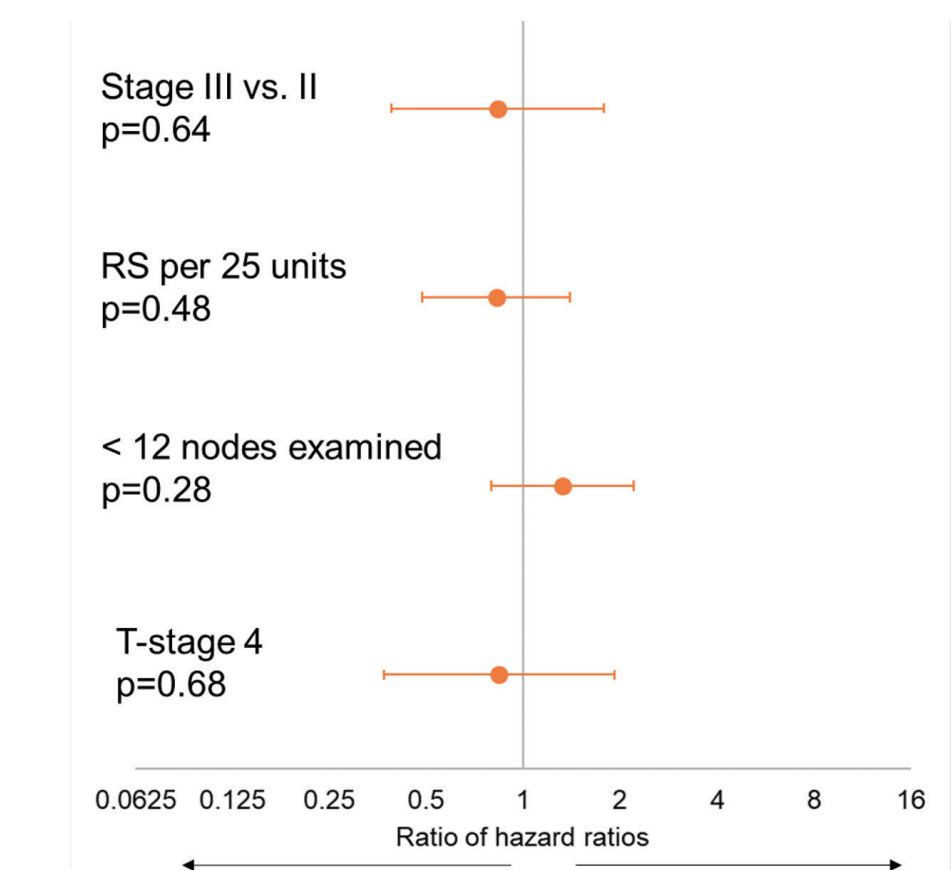

Lower HR with 5FU+oxaliplatin vs. 5FU Higher HR with 5FU+oxaliplatin vs. 5FU

Figure S4 Tests of interaction of treatment with other covariates in NSABP C-07. Ratio of HR: estimates and 95\% CI with Wald test P values. There were insufficient recurrence events among MMR-deficient patients to test for interaction. RS, 12-gene Oncotype DX Colon Recurrence Score result; HR, hazard ratios; 5FU, fluorouracil; CI, confidence intervals; MMR, mis-match repair. 


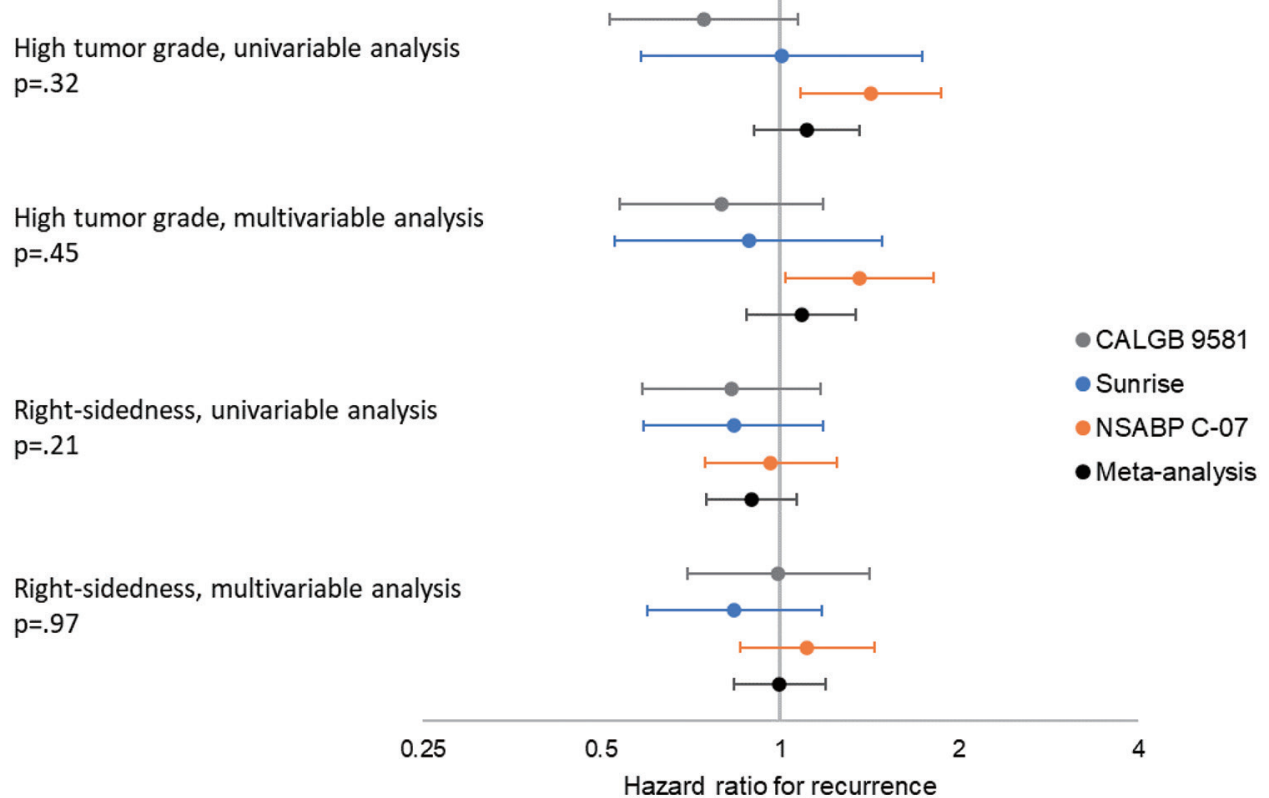

Figure S5 Meta-analysis estimates and 95\% CI for HR of high tumor grade and right-sided tumor location with recurrence: univariable analysis and multivariable together with stage, RS, number of nodes examined, T-stage, and MMR status; meta-analysis Wald test P values. CI, confidence intervals; HR, hazard ratios; RS, 12-gene Oncotype DX Colon Recurrence Score result; MMR, mis-match repair.

Table S1 Correlations among model factors

\begin{tabular}{lccccc}
\hline Factor & $<12$ nodes examined & T-stage 4 & MMR deficient & Stage IIIAB vs. II & Stage IIIC vs. II \\
\hline RS & -0.02 & 0.13 & 0.03 & 0.02 & 0.03 \\
$<$ 12 nodes examined & & 0.03 & -0.12 & 0.03 & -0.03 \\
T-stage T4 & & 0.08 & 0.00 & 0.01 \\
MMR deficient & & & -0.02 & -0.01 \\
\hline
\end{tabular}

MMR, mis-match repair.

Table S2 Exploratory analysis assessing the incremental prognostic value of right-sidedness tumor location

\begin{tabular}{|c|c|c|c|}
\hline Effect & CALGB $9581(n=690)^{*}, 162$ events & SUNRISE $(n=597)^{\star}, 202$ events & NSABP C-07 ( $n=892), 245$ events \\
\hline$<12$ vs. $\geq 12$ nodes examined & $1.12(0.79,1.59) ; P=0.53$ & $1.78(1.23,2.58) ; P=0.002$ & $1.50(1.16,1.95) ; P=0.002$ \\
\hline T-stage T4 vs. T3 & $1.04(0.51,2.13) ; P=0.92$ & $1.18(0.80,1.73) ; P=0.41$ & $2.70(1.80,4.04) ; P<0.001$ \\
\hline $\begin{array}{l}\text { MMR deficient vs. proficient/ } \\
\text { unknown }\end{array}$ & $0.62(0.39,1.01) ; P=0.056$ & $0.76(0.32,1.79) ; P=0.53$ & $0.28(0.12,0.63) ; P=0.002$ \\
\hline Stage IIIC vs. II & - & $5.29(3.39,8.27) ; P<0.001$ & $5.64(3.75,8.47) ; P<0.001$ \\
\hline Oxali + 5FU vs. 5FU & - & - & $0.80(0.62,1.03) ; P=0.084$ \\
\hline Right-sided tumor & $0.99(0.70,1.14) ; P=0.97$ & $0.84(0.60,1.17) ; P=0.30$ & $1.12(0.86,1.45) ; P=0.40$ \\
\hline
\end{tabular}

Cox proportional hazards regression model $\mathrm{HR}$ with $95 \% \mathrm{Cl}$ and Wald test $\mathrm{P}$ values. RS, 12-gene Oncotype DX Colon Cancer Recurrence Score result; MMR, mis-match repair; Oxali, oxaliplatin; HR, hazard ratios; Cl, confidence intervals.

(C) Journal of Gastrointestinal Oncology. All rights reserved. 
Table S3 Exploratory analysis assessing the incremental prognostic value of tumor grade

\begin{tabular}{|c|c|c|c|}
\hline Effect & CALGB $9581(n=690)^{\star}, 162$ events & SUNRISE $(n=597)^{\star}, 202$ events & NSABP C-07 ( $n=892), 245$ events \\
\hline RS per 25 units & $1.56(1.14,2.15) ; P=0.006$ & 1.97 (1.36, 2.84); $P<0.001$ & 1.67 (1.27, 2.19); P<0.001 \\
\hline$<12$ vs. $\geq 12$ nodes examined & $1.09(0.80,1.50) ; P=0.59$ & 1.85 (1.29, 2.65); $P=0.001$ & $1.49(1.15,1.92) ; \mathrm{P}<0.003$ \\
\hline T-stage T4 vs. T3 & $1.07(0.56,2.03) ; P=0.85$ & $1.19(0.81,1.75) ; P=0.38$ & 2.72 (1.81, 4.07); $\mathrm{P}<0.001$ \\
\hline $\begin{array}{l}\text { MMR deficient vs. proficient/ } \\
\text { unknown }\end{array}$ & $0.71(0.44,1.16) ; P=0.17$ & $0.73(0.31,1.72) ; P=0.47$ & $0.27(0.12,0.61) ; P=0.002$ \\
\hline Stage IIIC vs. II & - & $5.32(3.42,8.26) ; \mathrm{P}<0.001$ & 5.47 (3.65, 8.20); P<0.001 \\
\hline Oxali + 5FU vs. 5FU & - & - & $0.82(0.64,1.06) ; P=0.14$ \\
\hline High tumor grade & $0.80(0.54,1.18) ; P=0.26$ & $0.89(0.53,1.49) ; P=0.64$ & $1.36(1.09,1.81) ; P=0.037$ \\
\hline
\end{tabular}

Cox proportional hazards regression model HR with $95 \% \mathrm{Cl}$ and Wald test $\mathrm{P}$ values. RS, 12-gene Oncotype DX Colon Cancer Recurrence Score result; MMR, mis-match repair; Oxali, oxaliplatin; HR, hazard ratios; $\mathrm{Cl}$, confidence intervals. 